\title{
Species-specific field testing of Entamoeba spp. in an area of high endemicity
}

F. Heckendorn ${ }^{1,2}$, E. K. N'Goran ${ }^{2,3}$, I. Felger ${ }^{1}$, P. Vounatsou ${ }^{1}$, A. Yapi ${ }^{3}$, A. Oettli ${ }^{1}$, H. P. Marti ${ }^{1}$, M. Dobler ${ }^{1}$, M. Traoré ${ }^{2}$, K. L. Lohourignon ${ }^{3}$ and C. Lengeler ${ }^{1 \star}{ }^{i}$ Swiss Tropical Institute, Department of Public Health and Epidemiology, P.O. Box, CH-4002 Basel, Switzerland; ${ }^{2}$ Centre Suisse de Recherches Scientifiques, 01 B.P. 1303, Abidjan 01, Côte d'Ivoire; ${ }^{3}$ Laboratoire de Biologie Animale, URF Biosciences, Université de Cocody, 22 B.P. 582, Abidjan 22, Cote d'Ivoire

\begin{abstract}
Entamoeba histolytica has been separated in recent years into 2 morphologically identical species: the apathogenic $E$. dispar and the pathogenic $E$. histolytica, only the latter being pathogenic. Although various laboratory techniques allow discrimination between the 2 species there is a lack of field data about the suitability of available diagnostic tests for use in epidemiological studies and few epidemiological studies using species-specific diagnosis have been performed at community level in endemic areas, especially in sub-Saharan Africa. We conducted a repeated cross-sectional study of 967 schoolchildren in central Côte d'Ivoire to compare and evaluate light microscopy, 2 different antigen detection assays, and one polymerase chain reaction (PCR) assay. Microscopy and a non-specific antigen capture Entamoeba enzyme-linked immunosorbent assay (ELISA) were used for the primary screening of all children (time $\left.t_{0}\right)$. The prevalence of the $E$. histolytica/E. dispar species complex at $t_{0}$ was $18 \cdot 8 \%$ by single microscopical examination and $31.4 \%$ using the non-specific ELISA. Approximately 2 months after the initial screening, fresh stool specimens were collected on 2 consecutive days $\left(t_{1}\right.$ and $\left.t_{2}\right)$ from (i) all the children who were positive by microscopy at $t_{0}(n=182)$ and (ii) 155 randomly selected children who were negative at the primary screening. These samples were tested with a second antigen detection ELISA specific for $E$. histolytica $(n=238)$ and with a species-specific PCR assay $(n=193)$. The second and third examinations $\left(t_{1}\right.$ and $\left.t_{2}\right)$ revealed an additional 43 infections with the species complex $E$. histolytical $E$. dispar, so that the cumulative microscopical prevalence for $t_{1}$ and $t_{2}$ was $27.7 \%$. The overall prevalence of $E$. histolytica by species-specific ELISA antigen detection was low $(0.83 \%)$, while the prevalence of $E$. dispar was $15 \%$. When analysing only microscopically positive samples by PCR $(n=129)$, the ratio $E$. histolytica:E. dispar was very low $(1: 46)$, suggesting that the vast majority of Entamoeba infections in this area were apathogenic. Both species-specific tests performed well but the ELISA was easier to use for large-scale field screening.
\end{abstract}

Keywords: amoebiasis, Entamoeba dispar, Entamoeba histolytica, diagnosis, epidemiology, Côte d'Ivoire

\section{Introduction}

It is now generally accepted that there are 2 genetically distinct but morphologically indistinguishable species of Entamoeba, $E$. histolytica and $E$. dispar (see DIAMOND \& CLARK, 1993). Only $E$. histolytica has the potential to cause dysentery and extra-intestinal disease; $E$. dispar is considered to be a harmless commensal. The World Health Organization has reaffirmed the definition of amoebiasis as infection with $E$. histolytica sensu stricto, with or without clinical manifestation, and recommended that ' $E$. histolytica should be specifically identified and, if present, treated; if only $E$. dispar is identified, treatment is unnecessary' (ANONYMOUS, 1997)

The diagnosis of $E$. histolytica has traditionally relied upon microscopical examination of fresh or fixed stool specimens. A number of epidemiological studies had been performed before the clear distinction of the 2 species was established. Because they are microscopically indistinguishable, the validity of studies based on stool surveys only has been questioned. The frequently quoted statistics of 40 million to 50 million people infected and up to 100000 dying of amoebiasis worldwide are based in large part on these parasitological surveys, and this has led to an unclear picture of the epidemiology of amoebiasis (WALSH, 1986; ANONYMOUS, 1997).

In recent years, several alternative diagnostic methods have been developed for the differentiation of the 2 species: isoenzyme typing (SARGEAUNT et al., 1978, 1987), deoxyribonucleic acid (DNA) probes for use with the polymerase chain reaction (PCR) and restriction endonuclease pattern analysis (TANNICH \& BURCHARD, 1991; ROMERO et al., 1992; KATZWINKEL-WLADARSCH et al., 1994; TrOLl et al., 1997), and enzyme-linked immunosorbent assay (ELISA) antigendetection systems based on monoclonal antibodies

*Author for correspondence; phone +41 $61284 \quad 8221$, fax +41612717951, e-mail christian.lengeler@unibas.ch
(ABD-Alla et al., 1993; HAQUE et al., 1994). Cultivation of amoebae followed by isoenzyme analysis is a standard method of differentiation of $E$. histolytica and $E$. dispar. However, this method is time-consuming, not widely available and essentially impractical for large-scale epidemiological studies. PCR amplification of ribosomal ribonucleic acid (rRNA) genes of Entamoeba spp., as well as a new ELISA directed against the $\mathrm{Gal} / \mathrm{GalNAc}$ lectin (produced by TechLab Inc., Blacksburg, VA, USA) have been reported to approach the sensitivity and specificity of isoenzyme analysis in detecting $F$. histolytica directly in stool samples of symptomatic patients (HAQUE et al., 1995, 1998). However, a comparative study using cultured parasites as the source of DNA and antigen showed that the PCR was more sensitive than antigen detection by ELISA (MIRELMAN et al., 1997).

Given the current paucity of reliable data on the worldwide distribution of $E$. histolytica and $E$. dispar, it is critically important to arrive at better estimates of the burden of $E$. histolytica infection by using improved diagnostic tests. In sub-Saharan Africa only a few epidemiological surveys using species identification by isoenzyme analysis have been conducted, in South Africa (JACKSON et al., 1982; GATHIRAM \& JACKSON, 1985, 1987) and Ethiopia (GATTI et al., 1998). Information is crucial for the clinical and public health management of the disease, at both national and global levels. Moreover, adequate diagnostic methods and epidemiological information are urgently needed in view of the recent developments towards an amoebiasis vaccine (STANLEY, 1997; HUSTON \& PETRI, 1998). Obtaining further species-specific prevalence data on amoebiasis and the development of field testing strategies are therefore of high priority.

Based on previous work in Côte d'Ivoire revealing a high prevalence of the $E$. histolytica/E. dispar complex (UTZINGER et al., 1999), the objectives of the present study were (i) to assess the prevalence of the complex in children in Côte d'Ivoire using a newly available 
ELISA and repeated microscopy, (ii) to identify $E$. histolytica specifically using an established PCR assay (TROLL et al., 1997) and an antigen detection ELISA test, and (iii) to compare the diagnostic performance of these tests.

\section{Materials and Methods}

Study area and population surveyed

The study was carried out near the town of Agboville in the south of Côte d'Ivoire between March and June 2000. All schoolchildren attending standards $4-6$ at 8 randomly selected primary schools in the Agboville school district were enrolled. The objectives of the study were discussed with the school directors and, after obtaining their consent, the sex and age of the children were recorded. The day before the first survey, children were issued with a small plastic container and asked to return the containers with a small portion of their morning stool. After stool collection, children were issued with a new container for the following sample collection. In total 3 stool samples were collected, at time $t_{0}$ at the beginning of the study, and at times $t_{1}$ and $t_{2}$, approximately 2 months after $t_{0}$ (Fig. 1).

Identification of $\mathrm{E}$. histolytica/E. dispar complex infections

The stool specimens were taken to the laboratory in Abidjan within $3 \mathrm{~h}$ after collection. Before being further processed, each specimen was examined macroscopically for consistency with special emphasis on liquid specimens and on blood visible by eye.

A total of 967 stool samples was collected at time $t_{0}$, and a $1-2 \mathrm{~g}$ portion was preserved in sodium acetate-

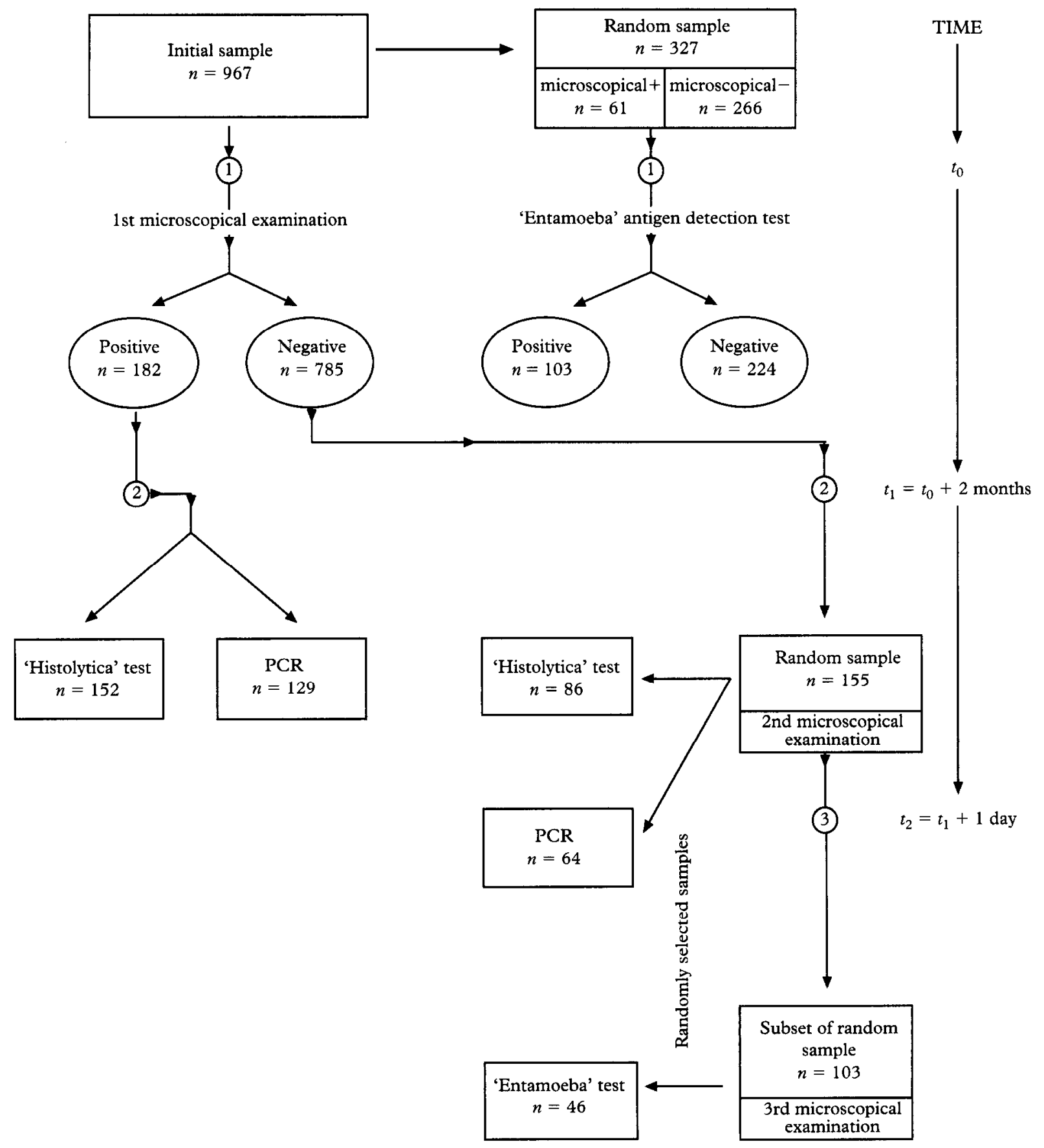

Fig. 1. Screening procedure for 967 schoolchildren in Côte d'Ivoire for detection of the Entamoeba histolyticalE. dispar complex and specific identification of the parasites. Numbers in circles indicate collection of fresh stool specimens. 
acetic acid-formalin (SAF). The samples were examined after processing according to MARTI \& ESCHER (1990) by light microscopy within 6 weeks (Fig. 1). All samples containing $E$. histolytica/E. dispar and other protists and helminths were recorded semi-quantitatively by distinguishing between 3 levels: $1+=1-5$ parasites seen per slide; $2+=1-2$ parasites seen per microscope field; $3+=$ more than 2 parasites per microscope field. Microscopical examination was done by one of 3 skilled and experienced laboratory technicians. Slides containing cysts or trophozoites of $E$. histolytica/ $E$. dispar were systematically checked and confirmed by a second technician.

A single Kato-Katz thick smear was also prepared from the stool samples collected at $t_{0}$, according to KATZ et al. (1972). The slides were examined by light microscopy and all helminth eggs were identified and recorded quantitatively.

Approximately 2 months after the first screening, 155 fresh stool samples were collected from a random selection of previously microscopically negative chil dren on 2 consecutive days (times $t_{1}$ and $t_{2}$ ), processed as described above and examined by light microscopy during the following 3 weeks. These 2 repeat examinations allowed the comparison of repeated microscopica examinations with the 'Entamoeba' ELISA. The results of examinations at $t_{0}$ and $t_{1} / t_{2}$ were not pooled for analysis because of the risk of new infections occurring during the 2 months interval between $t_{0}$ and $t_{1} / t_{2}$

\section{Antigen detection ELISA}

A randomly selected sample of 327 specimens of the initial 967 stool samples was tested at $t_{0}$ with the 'Entamoeba' antigen capture ELISA, to detect the Gal/ GalNAc lectin of both $E$. histolytica and $E$. dispar. Additionally, and for comparison with repeated microscopical samples, 46 of the initially microscopically negative specimens were also tested at $t_{1} / t_{2}$ by the 'Entamoeba' ELISA. The tests were performed according to the manufacturer's instructions (TechLab Inc.) and the optical density was measured with an automatic photometer at $450 \mathrm{~nm}$.

\section{Species-specific testing}

Species-specific diagnosis was done with the aid of (i) a PCR assay developed by TROLL et al. (1997) and (ii) the 'Histolytica' ELISA antigen capture test (TechLab Inc.) using stool specimens of children found to be infected with $E$. histolytica/E. dispar at the first microscopical examination $\left(t_{0}\right)$ and samples from randomly selected microscopically negative children (Fig. 1). Due to problems in tracing children and also sometimes to insufficient material in the samples, the numbers of specimens tested by the 2 specics-specific tests differed. The PCR targets rRNA genes of both $E$. histolytica and $E$. dispar in 2 separate reactions and therefore allows positive identification of both species. By contrast, the 'Histolytica' antigen detection ELISA is specific only for $E$. histolytica adhesin.

\section{PCR assay}

After initial concentration in SAF fixative the resulting pellets were washed 3 times with distilled water, transferred to $1.5 \mathrm{~mL}$ Eppendorf tubes, and suspended in $250 \mu \mathrm{L}$ digestion buffer $(100 \mathrm{mM}$ Tris, $\mathrm{pH} 8.0$; $25 \mathrm{~mm}$ ethylenediaminetetraacetic acid). The samples were then frozen and transported to Switzerland for further analysis. Subsequent analysis of the samples was done according to TROLL et al. (1993), with the following modifications: (i) DNA was purified after extraction using the QIAamp tissue mini-kit (Qiagen $A G$, Switzerland) in order to eliminate PCR inhibitors; (ii) the purified DNA was used without dilution in the PCR; (iii) deoxythymidine triphosphate was used instead of deoxyuridine triphosphate; (iv) restriction endonuclease (DraI) digests were performed on only a small number of $E$. dispar samples, in particular those showing faint bands, in order to confirm the identity of the amplified fragment.

\section{'Histolytica' antigen detection test}

In this assay $E$. histolytica is specifically identified due to differences in the $\mathrm{Gal} / \mathrm{GalNAc}$ lectin between $E$. histolytica and $E$. dispar (see HAQUE et al., 1995). The tests were performed according to the manufacturer's instructions (TechLab Inc.).

\section{Chemotherapy}

Treatment for intestinal helminth and protozoan parasites was given to all children found to be infected according to the Côte d'Ivoire national guidelines. Medication and doses used were approved by the Chief Medical Officer of the Agboville Health Department and administered by a pharmacist of the local Health Department. E. histolyticalE. dispar and Giardia duodenalis infections were treated with 3 daily doses of metronidazole $(10 \mathrm{mg} / \mathrm{kg}$ bodyweight) for $8 \mathrm{~d}$. Infections with Schistosoma mansoni were treated with a single dose of praziquantel ( $40 \mathrm{mg} / \mathrm{kg}$ bodyweight) and all other helminths (i.e. Ascaris lumbricoides, Trichuris trichiura, hookworms) with a single dose of albendazole.

All results of the microscopical examination and all data from the other diagnostic assays were doubleentered into the Excel ${ }^{\mathrm{TM}} 1997$ software package. After transfer to the Foxpro ${ }^{\mathrm{TM}} 2.6$ database, frequency and consistency tests were carried out. Analysis of associations and diagnostic performance calculations were carried out with the STATA ${ }^{\mathrm{TM}} 7.0$ statistics package (Stata Corporation, College Station, Texas, USA).

\section{Results}

Parasitology and non-specific ELISA

Complete results for the first microscopical examination were obtained for 967 children $(90 \%$ of the enrolled children). The median age was 12 years with a range from 8 to 16 years. There were significantly more boys $(n=589)$ than girls $(n=378) \quad(z=38 \cdot 8$, $P<0.001)$. Macroscopic stool examination revealed 574 liquid/unformed specimens (59\%). Only 5 children presented specimens containing macroscopically detectable blood, of which only one was subsequently shown to harbour $E$. histolytica/E. dispar by microscopy. There was no association between liquid/unformed specimens and infection with $E$. histolytica/E. dispar (odds ratio $=1 \cdot 13,95 \%$ confidence interval $(\mathrm{CI}) 0.82-$ $1 \cdot 56)$.

The point prevalence of $E$. histolyticalE. dispar for the single microscopical examination at $t_{0}$ was $18.8 \%(182 /$ $967 ; 95 \%$ CI 16.4-21.4). Most of the children infected with $E$. histolytica/ $E$. dispar showed infection levels of $1+(83 \%)$. The predominant protozoan parasite was $E$. coli with a prevalence of $63.4 \%$. Prevalences for all protozoa are presented in Table 1.

The combined results from the single Kato-Katz thick smear and the first microscopical examination showed that hookworms and $S$. mansoni were the commonest helminths (Table 1).

The non-specific 'Entamoeba' antigen detection ELISA was performed on a total of 327 specimen randomly selected from the initial 967 (Fig. 1). The parasitological prevalence of $E$. histolytica/E. dispar infections within this sample based on a single microscopical examination was $18.7 \%(61 / 327)$, which is close to the prevalence of $18.8 \%$ found in the overal sample of 967 children. The 'Entamoeba' ELISA detected 103 positive cases, giving a higher prevalence rate of $31 \cdot 5 \%(103 / 327 ; 95 \%$ CI $26 \cdot 5-36 \cdot 8)$.

Stool specimens of 155 children who were negative at $t_{0}$ werc collectcd again at $t_{1}$. At $t_{2}$, one day later, only 103 of them were present. These later examinations revealed an additional 43 infections with the $E$. histoly- 
Table 1. Prevalence of intestinal protozoa among 967 schoolchildren in Côte d'Ivoire screened by direct stool microscopy ${ }^{\mathrm{a}}$ and intestinal helminths among 885 children screened by direct microscopy ${ }^{a}$ and a single Kato-Katz thick smear

\begin{tabular}{lcc}
\hline Intestinal parasite & $\begin{array}{c}\text { No. of infected } \\
\text { children }\end{array}$ & Prevalence $(\%)^{\mathrm{b}}$ \\
\hline Protozoa & & \\
Entamoeba histolytica/Entamoeba dispar & 182 & $18 \cdot 8(16 \cdot 4-21 \cdot 4)$ \\
Entamoeba coli & 613 & $63 \cdot 4(60 \cdot 6-66 \cdot 8)$ \\
Giardia duodenalis & 77 & $8 \cdot 0(6 \cdot 3-9 \cdot 9)$ \\
Endolimax nana & 447 & $46 \cdot 2(43 \cdot 3-49 \cdot 7)$ \\
Chilomastix mesnili & 98 & $10 \cdot 1(8 \cdot 3-12 \cdot 3)$ \\
Entamoeba hartmanni & 126 & $13 \cdot 0(11 \cdot 0-15 \cdot 4)$ \\
Iodamoeba buetschlii & 85 & $8 \cdot 8(7 \cdot 1-10 \cdot 8)$ \\
Blastocystis hominis & 81 & $8 \cdot 4(6 \cdot 7-10 \cdot 4)$ \\
Helminths & 189 & $21 \cdot 4(18 \cdot 7-24 \cdot 2)$ \\
Schistosoma mansoni & 271 & $30 \cdot 6(27 \cdot 6-33 \cdot 8)$ \\
Hookworm & 78 & $8 \cdot 8(7 \cdot 0-10 \cdot 9)$ \\
Ascaris lumbricoides & 85 & $9 \cdot 6(7 \cdot 7-11 \cdot 7)$ \\
Trichuris trichiura & &
\end{tabular}

asing sodium acetate-acetic acid-formalin fixation (MARTI \& ESCHER, 1993).

${ }^{\circ} 95 \%$ Confidence interval in parentheses.

tica/E. dispar species complex; the cumulative prevalence for $t_{1}$ and $t_{2}$ was $27 \cdot 7 \%$.

\section{Species-specific testing}

152 and 129 samples which were positive at $t_{0}$ were tested with the specific 'Histolytica' ELISA and the PCR, respectively (Fig. 1). The set of samples tested by the 2 assays did not overlap completely due to problems in sample preparation. The 'Histolytica test' detected $3 / 152 \mathrm{E}$. histolytica infections $(2.0 \% ; 95 \%$ CI $0 \cdot 7-5 \cdot 6)$ and the PCR test $2 / 129(1 \cdot 6 \% ; 95 \%$ CI $0 \cdot 4-5 \cdot 5)$. E dispar was detected by the PCR in 93 of the 129 samples tested $(72 \cdot 1 \% ; 95 \%$ CI $63 \cdot 8-79 \cdot 1) .34$ of the 129 samples found to be positive by microscopy were negative by PCR $(26 \cdot 4 \% ; 95 \%$ CI $19 \cdot 5-34 \cdot 6)$. Based on PCR results with the microscopically positive samples, the species ratio $E$. histolytica: $E$. dispar was $c$. 1:46. No additional $E$. histolytica case was detected by the 'Histolytica' test or by the PCR when testing the microscopically negative samples.

Performance of diagnostic tests: non-specific tests

Using isoenzyme analysis as the reference test, the 'Entamoeba' antigen detection assay has been shown to be more sensitive $(80 \%$ vs. $60 \%$ ) and more specific (99\% vs. $79 \%$ ) than microscopy (HAQUE et al., 1995).
We therefore used the results of the 'Entamoeba' test to evaluate single and repeated microscopical examination (Table 2). Single microscopical examination of a stool sample collected at $t_{0}$ was moderately sensitive $(68.9 \%$; $95 \%$ CI $56 \cdot 4-79 \cdot 1)$ and specific $(77 \cdot 1 \% ; 95 \%$ CI $71 \cdot 7-81 \cdot 7)$. Repeated microscopical examination on 2 consecutive days at $t_{1}$ and $t_{2}$ improved sensitivity $(77.8 \% ; 95 \%$ CI $54.8-91 \cdot 0)$ and specificity $(89.3 \%$; $95 \% \mathrm{CI} 72 \cdot 8-96 \cdot 3)$, although this was measured on a much smaller sample ( $n=46$ compared with $n=327$ ), which is reflected in the much wider $95 \%$ confidence intervals. If we had taken single microscopy as reference standard, the sensitivity of the 'Entamoeba' test would have been only $40 \cdot 8 \%(95 \%$ CI $31 \cdot 8-50 \cdot 4)$ and the specificity would have been $91 \cdot 5 \%(95 \%$ CI $87 \cdot 1-$ $94 \cdot 5)$. With repeated microscopical examination as reference at $t_{1}$ and $t_{2}$, the sensitivity of the 'Entamoeba' test would have been $82.4 \%(95 \%$ CI $59 \cdot 0-93 \cdot 8)$ and the specificity would have been $86.2 \%(95 \%$ CI $69 \cdot 4-$ $94.5)$, again on a much smaller sample.

We also compared the 'Entamoeba' ELISA with the PCR, combining the PCR results for E. histolytica and $E$. dispar. In total, 101 specimens were examined by both PCR and the 'Entamoeba' test. PCR and the 'Entamoeba' test detected 75 and 53 E. histolytica/E. dispar infections, respectively (Fig. 2). The agreement

Table 2. Sensitivity and specificity of one and two consecutive microscopical stool examinations for the detection of the Entamoeba histolyticalE. dispar complex compared with the non-species-specific enzyme-linked immunosorbent assay

\begin{tabular}{|c|c|c|c|}
\hline \multirow[b]{2}{*}{ Microscopy } & \multicolumn{3}{|c|}{ ELISA $^{a}$} \\
\hline & Positive & Negative & Total \\
\hline \multicolumn{4}{|c|}{ Single examination ${ }^{\mathrm{b}}$} \\
\hline Positive & 42 & 61 & 103 \\
\hline Negative & 19 & 205 & 224 \\
\hline Total & 61 & 266 & 327 \\
\hline \multicolumn{4}{|c|}{ Two examinations ${ }^{b, c}$} \\
\hline Positive & 14 & 3 & 17 \\
\hline Negative & 4 & 25 & 29 \\
\hline Total & 18 & 28 & 46 \\
\hline
\end{tabular}

${ }^{2}$ Enzyme-linked inmmunusurbent assay for Encamoebu sp.

${ }^{b}$ Using sodium acetate-acetic acid-formalin fixation (MARTI \& ESCHER, 1993).

${ }^{\circ}$ On consecutive days, approximately 2 months after the single examination. 
A

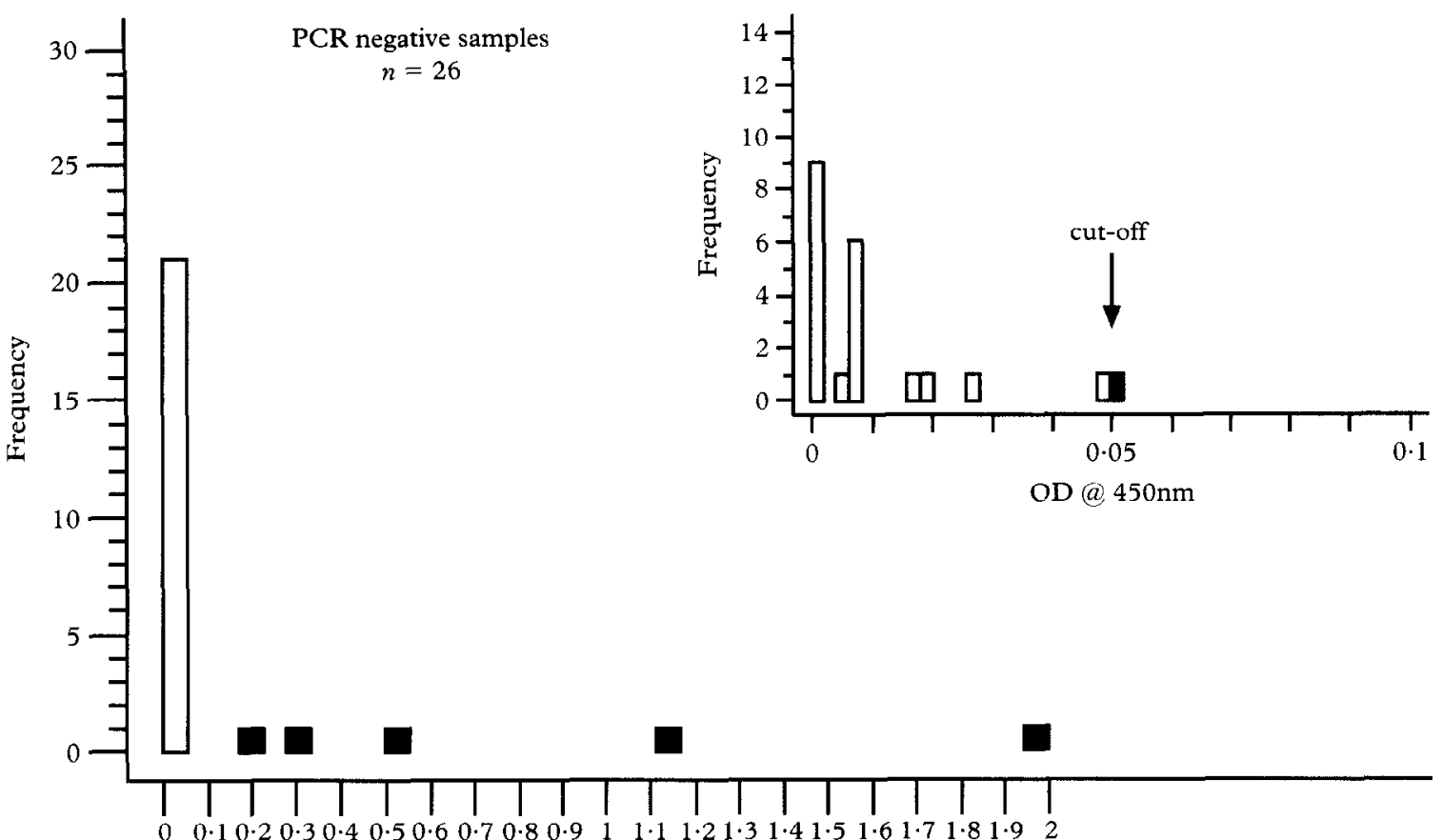

OD $@ 450 \mathrm{~nm}$

B

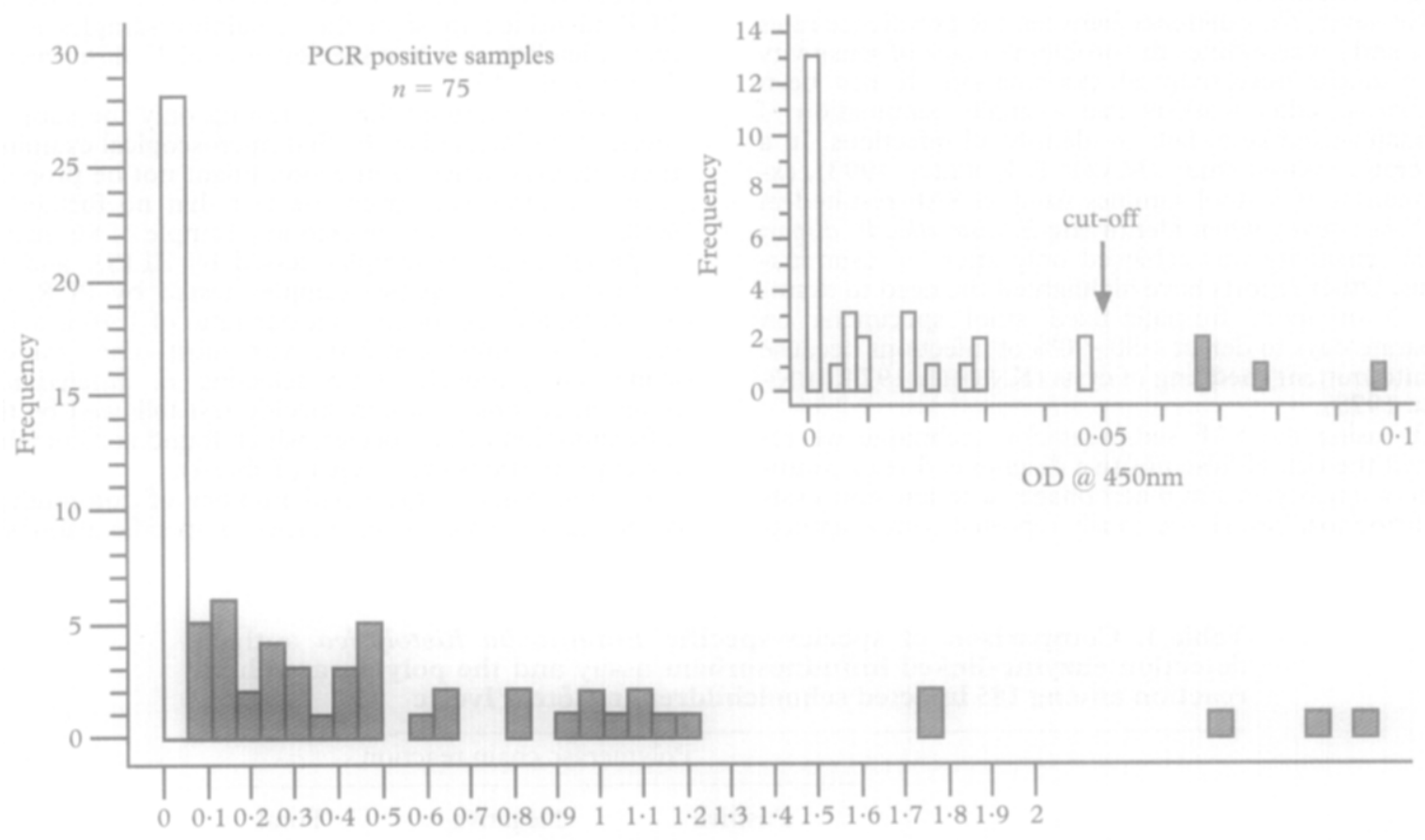

OD@450nm

Fig. 2. Optical density (OD) values for the Entamoeba enzyme-linked immunosorbent assay (ELISA) of stool specimens tested previously by the polymerase chain reaction (PCR): (A) 26 PCR-negative samples; (B) 75 PCR-positive samples. The small inserts show the same data for a narrower OD range and the OD positivity cut-off value of the Entamoeba $\operatorname{ELISA}(\mathrm{OD}=0.05)$; white bars indicate concordance between the PCR and ELISA results, shaded bars indicate discordance. 
of the 2 tests in detecting positives was only $46.5 \%$ $(95 \%$ CI $36 \cdot 5 \%-56 \cdot 7 \%)$.

\section{Performance of diagnostic tests: species-specific tests}

185 specimens were tested with both the 'Histolytica' antigen detection ELISA and the PCR. The microscopical prevalence of the $E$. histolytica/E. dispar complex in this sample was $76.8 \%(142 / 185 ; 95 \%$ CI $70.2-$ $82 \cdot 3)$. Both specific assays detected only a small number of $E$. histolytica infections; 3 by the specific antigen detection ELISA and 2 by the PCR (Table 3 ). Of the 3 $E$. histolvtica cases detected by ELISA, the PCR result agreed in one case and identified the remaining 2 specimens as containing $E$. dispar. The remaining positive sample detected by PCR was positive for both $E$. histolytica and $E$. dispar but was found to be negative by the 'Histolytica' antigen detection ELISA. As a quality control measure, the samples found to be positive for E. histolytica by either PCR $(n=2)$ or 'Histolytica' ELISA ( $n=3$ ) were re-examined by PCR by an independent investigator. The second examination confirmed all the results. Using the PCR as reference standard, the specific antigen detection ELISA exhibited an overall test efficiency (proportion of true positives and true negatives) of $98.4 \%$ (95\% CI 95.3 $99 \cdot 4)$.

\section{Discussion}

The high endemicity of $E$. histolytica/E. dispar in Côte d'Ivoire was confirmed by the present study with schoolchildren, who had an infection prevalence of $18.8 \%$ derived from a single microscopical examination and one of $31.4 \%$ with the 'Entamoeba' ELISA. The second and third microscopical examinations performed revealed a considerable number of additional infections but we did not consider these cumulatively because we could not exclude reinfections in the period of 2 months between the first and the second specimen collections. These prevalence estimates are conservative because of the probability of missing cases with a single examination

However, the difference between the prevalence rates at $t_{1}$ and $t_{2}$ exemplifies the problem of lack of sensitivity of a single microscopical examination. It has been shown by other workers that a single examination of formalin-fixed stool fails to identify all infections. In a laboratory-based study (MARTI \& KOELLA, 1993), examination of 3 stool samples fixed in SAF resulted in $92 \%$ sensitivity when identifying $E$. histolytica/ $E$. dispar $98 \%$ sensitivity was achieved only after $>4$ examinations. Other reports have highlighted the need to examine 3 or more formalin-fixed stool specimens on separate days to detect $>80-90 \%$ of infections because of intermittent shedding of cysts (KNIGHT, 1974; JUNIPER, 1978).

By using the SAF sedimentation technique we reduced the risk of false positive diagnoses due to confusion of leucocytes and macrophages with amoebic cysts and trophozoites, as previously reported with examina- tion of direct smears (KNIGHT, 1974). However, misidentification of other protozoa as $E$. histolyticalE. dispar cysts may have influenced the specificity of our detection. The risk of confounding immature $E$. coll cysts with those of $E$. histolytica/E. dispar is real, especially when differential morphological features such as chromatoid bodies are absent. This issue is of particular importance since both $E$. coli and $E$. histolytica/E. dispar are generally found in the same areas with poor hygienic conditions, and $E$. coli is usually the more prevalent species.

Other field-based studies in West Africa, relying on single examinations of formalin-ether concentrated stool specimens, also found high prevalences of $E$. histolyticalE. dispar $(52.3 \%$ and $37 \%$; BRAY \& HARRIS, 1977 and UTZINGER et al., 1999, respectively). In contrast, community-based surveys in other endemic areas, using similar diagnostic methods, reported substantially lower prevalence rates. A study in South Africa examined 800 specimens and found the prevalence of the species complex to be only $2 \cdot 8 \%$ (GATHIRAM \& JACKSON, 1985). In south-east Asia and in Mexico, $E$. histolytica/E. dispar infection rates of $12 \cdot 4 \%$ and $8 \cdot 1 \%$ have been reported (ACUNA-SoTO et al., 1993; RIVERA et al., 1998). In numerous other studies, prevalences of $E$. histolytica/E. dispar were mostly based on examination of direct smears and/or the surveys were hospital-based rather than community-based, and therefore cannot be compared easily with our findings.

This is the first community-based study looking at species-specific prevalence of $E$. histolytica and $E$. dispar in sub-Saharan Africa using diagnostic methods other than isoenzyme analysis. $E$. histolytica infections were assessed separately by a PCR assay and the 'Histolytica' ELISA antigen detection test developed by Techlab Inc. Among specimens shown microscopically to contain $E$. histolytica/E. dispar, both species-specific tests detected a surprisingly low number of $E$. histolytica infections ( 2 by the PCR assay, including one sample with both $E$. histolytica and $E$. dispar, and 3 by the nonspecific ELISA antigen detection test). As expected, PCR identified most of the remaining samples as $E$. dispar, leading to a low species ratio of $E$. histolytica to E. dispar of 1:46.

It could be argued that by testing only the subjects found to be infected at the first microscopical examination, the prevalence of infection might not be properly reflected. However, given the fact that no further $E$. histolytica case was discovered in a sample of 86 microscopically negative samples tested by ELISA and 64 microscopically negative samples tested by PCR, we are reasonably confident that our ratio of 1:46 is accurate. This finding contrasts with most other surveys using the approach of pre-selecting $E$. histolytica/E. dispar infections by a non-specific test followed by the differentiation of the species, which found considerably lower proportions of $E$. dispar (Table 4).

On the basis of the small number of communitybased studies which involved species-specific diagnosis,

Table 3. Comparison of species-specific Entamoeba histolytica antigen detection enzyme-linked immunosorbent assay and the polymerase chain reaction among 185 infected schoolchildren in Côte d'Ivoire

\begin{tabular}{lcrr}
\hline & \multicolumn{3}{c}{ Polymerase chain reaction } \\
\cline { 2 - 4 } & Positive & Negative & Total \\
\hline Species-specific ELISA & & & \\
Positive & 1 & 2 & 3 \\
Negative & $1^{\mathrm{b}}$ & 181 & 182 \\
Total & 2 & 183 & 185 \\
\hline
\end{tabular}

a Enzyme-linked immunosorbent assay

'Specimen positive for both Entamoeba histolytica and E. dispar. 
Table 4. Reported prevalence rates and ratios for Entamoeba histolytica and E. dispar in amoebiasis endemic areas

\begin{tabular}{|c|c|c|c|c|}
\hline Area & Reference & $\begin{array}{l}\text { Diagnostic } \\
\text { method }^{a}\end{array}$ & Prevalence $(\%)^{\mathrm{b}}$ & Species ratio ${ }^{c}$ \\
\hline Turkey & JETTER et al., 1997 & PCR & $31 \cdot 6 \quad(73 / 231)$ & E. dispar only \\
\hline South Africa & JACKSON et al., 1982 & Isoenzymes & $10 \cdot 0(139 / 1381)$ & $1: 10$ \\
\hline Mexico & ACUNA-SOTO et al., 1993 & PCR & $12 \cdot 4 \quad(25 / 201)$ & Note $^{d}$ \\
\hline Philippines & RIVERA et al., 1998 & PCR & $8 \cdot 1(152 / 1872)$ & $1: 8 \cdot 5$ \\
\hline Bangladesh & HAQUE et al., 1997 & ELISA & $8.0 \quad(79 / 987)$ & $1: 8$ \\
\hline Bangladesh & HAQUE et al., 1998 & ELISA & $17 \cdot 4(118 / 680)$ & $1: 2 \cdot 7$ \\
\hline Côte d'Ivoire & This study & ELISA \& PCR & $31 \cdot 5(103 / 327)$ & $1: 46$ \\
\hline
\end{tabular}

${ }^{a}$ ELISA $=$ enzyme-linked immunosorbent assay; $\mathrm{PCR}=$ polymerase chain reaction.

${ }^{\mathrm{b}} E$. histolytica/E. dispar complex.

${ }^{\mathrm{C}} E$. histolytica: $E$. dispar.

d $56 \%$ of all individuals infected harboured mixed infections with $E$. histolytica and $E$. dispar.

it appears that the species ratio $E$. histolytica:E. dispar varies considerably. Whether this observation reflects small-scale rather than large-scale geographical differences in the distribution of $E$. histolytica cannot be answered at this time. In order to increase the epidemiological understanding of amoebiasis, this issue needs urgently to be further investigated.

The 'Entamoeba' test and the PCR assay showed only limited agreement in detecting infections $(46 \cdot 5 \%)$. PCR detected a substantially higher number of $E$. histolyticalE. dispar infections than the 'Entamoeba test'. Possibly the additional cases detected by PCR reflected the low sensitivity of a single examination using the other test. However, technical problems with the PCR technique cannot simply be excluded. This technique is potentially prone to cross-contamination due to its power to amplify minute traces of target DNA. To test for possible contamination, our samples were retested twice by PCR by an independent investigator using additional controls, but we could not find a problem with the assay. However, contamination can occur between the field and the laboratory during sample handling or DNA preparation and this cannot be traced later.

When the PCR assay was taken as the standard, the 'Histolytica' ELISA antigen detection test showed high efficiency $(98 \cdot 4 \%)$. However, the 2 tests disagreed with 3 samples which were positive for $E$. histolytica by either PCR or ELISA (Table 3). In contrast, another study also using the TechLab specific ELISA and a PCR assay for the diagnosis of $E$. histolytica in stool samples, found a correlation of $84 \%$ between the 2 tests (HAQUE et al., 1998). Our findings concerning $E$. histolytica infections are difficult to interpret because of the small number of infections. Despite a rather large initial sample size we failed to detect many such infections because of the unexpectedly low $E$. histolytica/E. dispar ratio.

In conclusion, this study produced valuable data in relation to the occurrence of $E$. dispar and $E$. histolytica in communities in sub-Saharan Africa. While the prevalence of the species complex was high, the prevalence of $E$. histolytica was low. This might explain the paradox of the low amount of amoebiasis morbidity reported by health services on the African continent despite a high apparent microscopical infection rate. Field testing with the new generation of ELISA kits proved to be clearly superior to microscopy. Because of the small number of $E$. histolytica cases in our sample the intended comparison of test performance between the 'Histolytica' ELISA and the PCR did not result is a clear conclusion about which test is superior. However, unlike the PCR, the ELISA proved to be easy to use in the field and, if its high diagnostic performance is confirmed, it might well become the standard technique for large-scale field screening.

\section{Acknowledgements}

The present work was mainly supported by funds from the Swiss Tropical Institute in Basel. Additional support was provided by TechLab Inc. through supplying free kits for ELISA and PCR testing. The support of the Centre Suisse de Recherche Scientifique en Côte d'Ivoire, and especially its Director Dr Olivier Girardin, is gratefully acknowledged. Special thanks also go to the Agboville school district officer and all the teachers and pupils who participated in the study. The contribution of Mr Silue Kouna, who helped in the laboratory, is gratefully acknowledged.

\section{References}

Abd-Alla, M. D., Jackson, T. F., Gathiram, V., El Hawey, A. M. \& Ravdin, J. I. (1993). Differentiation of pathogenic Entamoeba histolytica infections from non-pathogenic infections by detection of galactose-inhibitable adherence protein antigen in sera and faeces. Fournal of Clinical Microbiology, 31, 2845-2850.

Acuna-Soto, R., Samuelson, J., De Girolami, P., Zarate, L., Millan-Velasco, F., Schoolnick, G. \& Wirth, D. (1993). Application of the polymerase chain reaction to the epidemiology of pathogenic and non-pathogenic Entamoeba histolytica. American foumal of Tropical Medicine and Hygiene, 48, $58-70$.

Anonymous (1997). A consultation with experts on amoebiasis. Mexico City, Mexico, 28-29 January. WHO/PAHO/ UNESCO report. Epidemiological Bulletin, 18, 13-14.

Bray, R. S. \& Harris, W. G. (1977). The epidemiology of infection with Entamoeba histolytica in The Gambia, West Africa. Transactions of the Royal Society of Tropical Medicine and Hygiene, 71, 401-407.

Diamond, L. S. \& Clark, C. G. (1993). A redescription of Entamoeba histolytica Schaudinn, 1903 (emended Walker, 1911) separating it from Entamoeba dispar Brumpt, 1925. fournal of Eukaryotic Microbiology, 40, 340-344.

Gathiram, V. \& Jackson, T. F. (1985). Frequency distribution of Entamoeba histolytica zymodemes in a rural South African population. Lancet, i, 719-721.

Gathiram, V. \& Jackson, T. F. (1987). A longitudinal study of asymptomatic carriers of pathogenic zymodemes of Entamoeba histolytica. South African Medical Foumal, 72, 669-672.

Gatti, S., Mahdi, R., Bruno, A., Cevini, C. \& Scaglia, M (1998). A survey of amoebic infection in the Wonji area of central Ethiopia. Annals of Tropical Medicine and Parasitology, 92, 173-179.

Haque, R., Neville, L. M., Wood, S. \& Petri, W. A., jr (1994) Detection of Entamoeba histolytica and Entamoeba dispar directly in stool. American foumal of Tropical Medicine and Hygivie, 50, 595-596.

Haque, R., Neville, L. M., Hahn, P. \& Petri, W. A., jr (1995) Rapid diagnosis of Entamoeba infection by using Entamoeba and Entamoeba histolytica stool antigen detection kits. Fournal of Clinical Microbiology, 33, 2558-2561.

Haque, R., Faruque, A. S. \& Petri, W. A. (1997) Entamoeba histolytica and Entamoeba dispar infection in children in Bangladesh. Archives of Medical Research, 28, special number, 317-318.

Haque, R., Ali, I. K., Anther, S. \& Petri, W. A., jr (1998) Comparison of PCR, isoenzyme analysis, and antigen detection for diagnosis of Entamoeba histolytica infection. foumal of Clinical Microbiology, 36, 449-452. 
Huston, C. D. \& Petri, W. A., jr (1998). Host-pathogen interaction in amoebiasis and progress in vaccine development. European foumal of Clinical Microbiology and Infectious Diseases, 17, 601-614.

Jackson, T. F., Sargeaunt, P. G., Williams, I. E. \& Simjee, A E. (1982). Observations on zymodeme studies of Entamoeba histolytica in Durban, South Africa. Archives of Investigational Medicine (Mexico), 13, supplement 3, 83-88.

Jetter, A., Walderich, B., Britten, D., Mete, O., Goral, V. Burchard, G. D. \& Ackers, J. (1997). An epidemiological study of Entamoeba histolytica and Entamoeba dispar infection in eastern Turkey using a colorimetric polymerase chain reaction. Archives of Medical Research, 28, special number, 319-321.

Juniper, K. (1978). Amoebiasis. Clinical Gastroenterology, 7, $3-29$

Katz, N., Chaves, A. \& Pellegrino, J. (1972). A simple device for quantitative stool thick-smear technique in schistosomiasis mansoni. Revista do Instituto de Medicina Tropical de São Paulo, 14, 397-400.

Katzwinkel-Wladarsch, S., Loscher, T. \& Rinder, H. (1994) Direct amplification and differentiation of pathogenic and non-pathogenic Entamoeba histolytica DNA from stool specimens. American foumal of Tropical Medicine and Hygiene, 51, 115-118.

Knight, R. (1974). Amoebiasis. Tropical Doctor, 4, 6-11.

Marti, H. P. \& Escher, E. (1990). SAF-an alternative fixation solution for parasitological stool specimens. Schweizerische Medizinische Wochenschrift, 120, 1473-1476.

Marti, H. \& Koella, J. C. (1993). Multiple stool examinations for ova and parasites and rate of false-negative results. foumal of Clinical Microbiology, 31, 3044-3045.

Mirelman, D., Nuchamowitz, Y. \& Stolarsky, T. (1997). Comparison of use of enzyme-linked immunosorbent assaybased kits and PCR amplification of rRNA genes for simultaneous detection of Entamoeba histolytica and Entamoeba dispar. Fournal of Clinical Microbiology, 35, 2405-2407.

Rivera, W. L., Tachibana, H. \& Kanbara, K. (1998). Application of the polymerase chain reaction (PCR) in the epidemiology of Entamoeba histolytica and Entamoeba dispar infections. Tokai foumal of Experimental and Clinical Medicine, 23, 413-415.
Romero, J. L., Descoteaux, S., Reed, S., Orozco, E., Santos, J. \& Samuelson, J. (1992). Use of polymerase chain reaction and nonradioactive DNA probes to diagnose Entamoeba histolytica in clinical samples. Archives of Medical Research, 32, 277-279.

Sargeaunt, P. G., Williams, J. E. \& Grene, J. D. (1978). The differentiation of invasive and non-invasive Entamoeba histolytica by isoenzyme electrophoresis. Transactions of the Royal Society of Tropical Medicine and Hygiene, 72, 519-521.

Sargeaunt, P. G., Jackson, T. F., Wiffen, S., Bhojnani, R., Williams, J. E., Felmingham, D., Goldmeir, D., AllasonJones, E., Mindel, A \& Phillips, E. (1987). The reliability of Entamoeba histolytica zymodemes in clinical laboratory diagnosis. Archives of Investigational Medicine (Mexico), 18 , $69-75$.

Stanley, S. L. (1997). Progress towards development of a vaccine for amebiasis. Clinical Microbiological Reviews, 10 $637-649$.

Tannich, E. \& Burchard, G. D. (1991). Differentiation of pathogenic from non-pathogenic Entamoeba histolytica by restriction fragment analysis of a single gene amplified in vitro. Fournal of Clinical Microbiology, 29, 250-255.

Troll, H., Marti, H. P. \& Weiss, N. (1997). Simple differential detection of Entamoeba histolytica and Entamoeba dispar in fresh stool specimens by sodium acetate-acetic acid-formalin concentration and PCR. Fournal of Clinical Microbiology, $35,1701-1705$

Utzinger, J., N'Goran, E. K., Marti, H. P, Tanner, M. \& Lengeler, C. (1999). Intestinal amoebiasis, giardiasis and geohelminthiases: their association with other intestinal parasites and reported intestinal symptoms. Transactions of the Royal Society of Tropical Medicine and Hygiene, 93, $137-141$.

Walsh, J. A. (1986). Problems in recognition and diagnosis of amebiasis: estimation of the global magnitude of morbidity and mortality. Reviews of Infectious Diseases, 8, 228-238.

Received 14 November 2001; accepted for publication 1 March 2002

\section{Announcement}

\section{ROYAL SOCIETY OF TROPICAL MEDICINE AND HYGIENE Garnham Fellowships}

Professor Cyril Garnham was one of the UK's leading parasitologists in the 20th century and his work was characterized by outstanding achievement as both laboratory scientist and field worker in the tropics. The special place that Garnham occupies among his colleagues is recognized by the Fund set up in his memory to establish research fellowships for young scientists.

The aim of the Garnham Fellowship is to encourage young scientists to carry out short-term field projects. Suitable applicants are invited to apply to the Fund, which is administered by the Royal Society of Tropical Medicine and Hygiene.

There are no restrictions by nationality or age, and fellowship of the Royal Society of Tropical Medicine and Hygiene is not a requirement. Applications from non-Fellows should be supported by a Fellow who can attest to the value of the project and to the competence of the applicant to carry out the work.

- One Garnham Fellowship of up to $£ 2000$ will be awarded annually

- The Garnham Fellowship is to be used for short-term field projects of up to 2 years' duration

- Preference will be given to topics in parasitology or medical entomology and to applicants with less than 5 years' postdoctoral experience

- Applicants are required to submit a detailed project, with costing of the work proposed, and a supporting statement from their head of department or supervisor, at least 6 months before the date of commencement

- A short report should be submitted within 3 months of completion of the study

Application forms may be obtained from the Administrator, Royal Society of Tropical Medicine and Hygiene, Manson House, 26 Portland Place, London, W1B 1EY, UK; fax +44(0)20 7436 1389, e-mail mail@rstmh.org The closing date for receipt of applications is 15 September annually. 